\title{
Dietary and Lifestyle Factors Associated with Colorectal Cancer Risk and Interactions with Microbiota: Fiber, Red or Processed Meat and Alcoholic Drinks
}

\author{
Juan Tuan Ying-Xuan Chen \\ Division of Gastroenterology and Hepatology, Ren Ji Hospital, Shanghai Jiao Tong \\ University School of Medicine, Shanghai Institution of Digestive Disease, Shanghai, China
}

\section{Key Words}

Alcoholic drinks · Colorectal cancer · Fiber · Prevention · Red or processed meat

\begin{abstract}
Background: Diets and lifestyles have been strongly associated with colorectal cancer (CRC). In the past several decades, emerging evidence has suggested that the gut microbiota may have a role in the development of CRC. Its interaction with diets and lifestyles could affect the carcinogenesis of CRC. Summary: This review presents the most recent epidemiologic and experimental evidence of three factors that may convincingly have a role in CRC, including fiber, red or processed meat, and alcohol, focusing on potential mechanisms and their interactions with the gut microbiota. Key Message: High consumption of fiber, low consumption of red or processed red meat as well as minimizing alcohol intake have been associated with a lower risk of CRC. Many microbial metabolites formed from those three substances may mediate the microbial diversity and the composition and abundance of the gut microbiota, which eventually affects the balance between health and disease, including CRC. Practical Implications: Based on our synthetic review, clinicians may probably offer some recommendations and explanations to their patients who may want to modulate their diet and lifestyle to prevent CRC. As an easily modifiable environmental factor, it may be possible that applying dietary or lifestyle intervention could effectively protect against the development of CRC in the future.

(c) 2015 S. Karger AG, Basel
\end{abstract}




\section{Introduction}

As the third most common cancer and the fourth leading cause of cancer death in the world, colorectal cancer (CRC) caused about 694,000 deaths in 2012, with over 1.4 million new cases diagnosed globally [1], and its incidence rate is rapidly rising in less developed regions, with a poorer prognosis compared to that in more developed regions. Although routine screening reduces the incidence and mortality of CRC, its availability has been limited, particularly for subjects of low socioeconomic status. Although the etiology of this highly lethal disease remains unclear, numerous researchers have shown that many environmental factors play a vital role in an individual's risk of CRC. For these reasons, the global burden of CRC could be reduced by applying effective prevention measures such as dietary and lifestyle modifications and by the use of early detection tests.

Numerous epidemiologic and experimental investigations have shown that dietary modifications, nutritional or drug supplements and exercise were associated with a lower risk of CRC. However, most lifestyle studies were observational, gathering information from interviews or questionnaires to assess whether there is a link between such factors as daily diet and CRC, and the results were inconsistent or equivocal. The role of diets and lifestyle in human health and disease is complex, such as the dual-modulator role of folate [2].

There is growing concern that diet-induced human gut microbial changes are now supposed to cause growing epidemics of CRC. There are trillions of microorganisms residing in the human gut. Over the past decade, there was also emerging experimental evidence pointing to a link between gut microbiota and CRC, through comparing gut microbiota from people with and without CRC or from animal model. Gut microbiotas were composed of the four bacterial phyla (Firmicutes, Bacteroidetes, Proteobacteria and Actinobacteria) and a few other bacterial phyla. The composition and abundance of gut microbiota shift before or during the development of CRC and would be influenced by dietary patterns [3,4]. Studies have confirmed the association of variations in proportions of Bacteroidetes and Firmicutes with CRC. In a study comprehensively surveying and comparing fecal gut microbiota between 47 CRC cases and 94 matched controls, decreased bacterial diversity, enrichment of the phylum Bacteroidetes and depletion of Firmicutes (including Gram-positive, fiber-fermenting Clostridia) were found in cases [5]. In addition, the proinflammatory genera Fusobacterium and Porphyromonas were increased in CRC cases, which contributed to colitis and may be related to CRC risk [5]. The composition of the gut microbiome could be modulated by diet. Three predominant variants, or 'enterotypes', were proposed recently, namely Bacteroides, Prevotella and Ruminococcus. Studies in long- or short-term diet provide insights into the effects of the diet on intestinal microbiota. Long-term diet with protein and animal fat was dominated by Bacteroides, while diet with carbohydrates was dominated by the Prevotella enterotype [3]. In addition, short-term macronutrient change altered microbial community composition, and the animal-based diet increased the levels of bile-tolerant microorganisms and lowered Firmicutes, which metabolize dietary plant polysaccharides [6].

A better understanding of the mechanism involved in diet- and lifestyle-microbiota-host interaction will provide a potential strategy for the prevention of CRC. In this review, we summarize three factors that may convincingly have a role in CRC - fiber, red or processed meat and alcohol - and focus on their interactions with the gut microbiota.

\section{Fiber}

Through a systematic review of diet and cancer, the Colorectal Cancer 2011 Report showed that there is convincing evidence for decreased CRC risk with increased intake of total dietary fiber [7]. This concept has been in existence for more than decades, and extensive 
case-control and cohort studies have evaluated the relation between CRC and fiber. For example, a large prospective study in the European Prospective Investigation into Cancer and Nutrition (EPIC) indicated an approximately 40\% lower risk of CRC in the highest vs. lowest quintile of fiber intake [8]. However, another large cohort study showed a nonexistent association between dietary fiber intake and CRC (relative risk [RR] for the highest vs. the lowest quintile of fiber intake $=0.99,95 \%$ confidence interval [CI] $=0.85-1.15$ ) [9]. A prospective case-control study nested within seven UK cohort studies found that the multivariableadjusted odds ratio of CRC for the highest vs. the lowest quintile of fiber intake was 0.66 (95\% $\mathrm{CI}=0.45-0.96$ ) using data ascertained by food diaries. While using dietary data obtained by food frequency questionnaires, the association became statistically nonsignificant [10]. Though the results of these studies are inconsistent, a meta-analysis stated an approximately $10 \%$ lower risk of colorectal adenoma (CRA) per $10 \mathrm{~g} /$ day increase in fiber [11]. Recently, a large prospective study within a population-based screening trial pointed out that elevated total dietary fiber intake have reduced risks of incident CRA and distal colon cancer, but not of recurrent adenoma and CRC overall [12]. Based on these results and the benefit for other systems such as cardiovascular disease [13], a high-fiber diet can reasonably be recommended.

Substantial experimental evidence also supported the hypothesis that dietary fiber intake is associated with a low risk of CRC. One of the proposed mechanisms is bacterial fermentation of resistant starch to short-chain fatty acids (SCFAs), particularly butyrate. The process of fermenting fibers to SCFAs needs intestinal bacteria because germ-free mice produce almost no SCFAs [14]. SCFAs influence inflammation factors, tumor necrosis factor $\alpha$ and interleukin 6 for instance, and suppress the transcription factor nuclear factor $\kappa B$. The colonic epithelium selectively absorbs butyrate, and it can be further metabolized to generate ATP, the preferred energy source for colonocytes. Further, butyrate plays crucial roles in the cellular homeostasis of the colonic epithelium. That is to say, it acts as a histone deacetylase inhibitor, directly affecting gene expression, and has shown anti-carcinogenic, anti-inflammatory and anti-oxidant functions [15]. It also has an anti-cancer effect on cancer cells, enhancing apoptosis and suppressing proliferation [16].

Recently, the concept that diet affects human health partly by modulating gut microbiome composition has been proposed. Diet high in fiber vs. diet high in fat showed inverse associations with microbial taxa. Compared with extreme plant-based diets (high levels of fiber), the abundance of Bacteroidetes increased and several members of the butyrateproducing Firmicutes decreased in the animal-based diets (no fiber and high levels of fat and protein) [6]. Also, most volunteers on the resistant starch diet but not the non-starch polysaccharide diet showed increased levels of specific bacterial taxa such as Ruminococcus bromii and Eubacterium rectale [17]. In view of the result that the represented enterotype shifted from Ruminococcus in a greater percentage of healthy controls to Bacteroides in most carcinoma and adenoma patients [4] and that CRC cases tended to have depletion of fiberfermenting Clostridia [5], this could indirectly demonstrate the association between highfiber diet and low risk of CRC. Microbiome composition could alter detectably within $24 \mathrm{~h}$ of initiating a high-fat/low-fiber or low-fat/high-fiber diet through a controlled feeding study, but enterotype identity did not shift stably during the 10-day study, implying that the longterm diet may modulate enterotype clustering to improve health [3]. By comparing the fecal microbiota of European children who eat a diet high in animal protein and fat and that of rural African children from Burkina Faso, where the diet is high in fiber content, investigators found that the SCFA-producing bacteria were significantly more abundant in the Burkina Faso children, with a unique enrichment in the genera Prevotella and Xylanibacter which contain a set of bacterial genes for fiber hydrolysis, completely lacking in the European children. Overall, greater microbial richness and more SCFAs were also found in Burkina Faso 
than in the European children [18]. Furthermore, diets higher in fruits, vegetables and fiber have been shown to increase microbial richness at the gene and taxonomic level, which was associated with human health, whereas lower bacterial richness was associated with obesity, insulin resistance, metabolic syndrome and inflammatory disorders, all of which increase the risk of CRC [19].

Therefore, diet high in fiber, as described above, could on the long run maintain a stable and healthy gut community possessing high microbial diversity and low pathogen abundance, increasing butyrate-producing bacteria, promoting fiber hydrolysis, providing energy for colonocytes and possessing potential anti-inflammatory properties, eventually playing a protective role against CRC.

\section{Red or Processed Meat}

Red or processed meat as a contributing factor in CRC development has been verified in many epidemiologic studies. However, there are still debates on the potential relationship between red meat consumption and CRC [20,21]. Though some studies found no association between red or unprocessed meat and CRC, high processed meat intake was related with an increased risk of CRC, especially distal colon cancer [21, 22]. In the Colorectal Cancer 2011 Report [7] experts judged that red meat is a convincing cause of CRC, and some meta-analyses also concluded that an increased intake of red or processed meat was associated with CRC and CRA, the precursor of CRC $[23,24]$. In addition, the association was also pronounced between greater red or processed meat intake before CRC diagnosis and higher risk of death as a result of all causes among CRC patients [25].

Biologic mechanisms relating red and in particular processed red meat to CRC have been proposed in substantial experimental studies. Red or processed meat may contribute to CRC through metabolization of mutagens, such as polycyclic aromatic hydrocarbons, heterocyclic amines and dietary N-nitroso compounds. Besides, the catabolism of heme could further lead to the endogenous production of these compounds, which could initiate carcinogenesis through lipid peroxidation [26]. Generated secondary bile acids and end products of heme cause inflammation and cytotoxic effects, resulting in the formation of reactive nitrogen species/reactive oxygen species and cytokines, promoting epithelial cell hyperproliferation. Endogenous and exogenous $\mathrm{N}$-nitroso compound production increases through adding nitrates and nitrites to processed meats, enhancing the cytotoxicity of nitrosyl heme and reactive chlorine species, which may offer part of the explanation for why processed meat has been associated more strongly with the risk of CRC [27].

Besides the fact that those carcinogenic compounds are produced after metabolization, the gut microbiota also plays an important role within the process of CRC development related with red or processed red meat. As mentioned before, the Bacteroides enterotype was significantly increased in a majority of CRC and CRA patients. Indeed, several studies have demonstrated that the Bacteroides enterotype was highly associated with animal proteins and saturated fats, suggesting that high levels of protein and fat consumption, as in a Western diet, were characterized by this enterotype $[3,18]$. Moreover, diets rich in red meat promote the growth of sulfate-reducing bacteria that produce hydrogen sulfide, a genotoxic agent [19]. A positive association was also found between consumption of red meat and carcinoma-enriched bacteria such as Bacteroides massiliensis, Alistipes finegoldii and Bilophila wadsworthia [4]. Carcinoma-enriched metagenomic linkage groups (MLGs), including mlg-84, mlg-850 and mlg-1738, showed a positive correlation with a greater total meat or red meat consumption. These results were consistent with the Kyoto Encyclopedia of Genes and Genomes (KEGG) orthology (KO) analysis. Compared with adenoma, carcinoma 
abounded in $\mathrm{KO}$ modules for transporting the amino acids arginine, histidine and lysine, but not in those for amino acids synthesis [4]. Together, these results suggest that there was an enhanced availability of amino acids along with the adenoma-carcinoma development, which may be due to increasing amino acids-utilizing bacteria with long-term high red meat diet [4].

\section{Alcohol}

Most prospective cohort and case-control studies, but not all, indicate that high intake of alcohol increases the risk of CRC, although several data have shown either inconsistent or only modest associations [28]. A recent prospective cohort study from 12 countries at different economic levels in five continents reported that current drinking was associated with increased alcohol-related cancers (hazard ratio $=1.51,95 \% \mathrm{CI}=1.22-1.89$ ), including CRC [29]. A comprehensive dose-response meta-analysis also corroborated these findings, the RR for heavy drinkers compared with non-drinkers and occasional drinkers being 1.44 for CRC [30]. However, moderate alcohol consumption ( $<30 \mathrm{~g} /$ day) was not significantly associated with the incidence of CRC, and other factors, including a Mediterranean diet, folate deficiency and genetic susceptibility, would convert this association [31]. Nevertheless, another meta-analysis of light alcohol drinking (up to 1 drink/day) and cancer also showed no association for CRC, but an increasing risk of oral cavity, pharynx, esophagus and breast cancer [32]. The Korean Multi-center Cancer Cohort study found that a correlation between alcohol consumption and elevated risk of CRC existed in men, but not women [33]. The Colorectal Cancer 2011 Report also regarded alcoholic drinks as convincingly increasing the risk of CRC for men, and alcoholic drinks as probably increasing the risk for women [7]. This more elevated risk for men may be due to the generally higher consumption of alcohol among men, different preference in types of alcoholic drinks or hormone-related differences in alcohol metabolism [7].

The mechanisms of the carcinogenic effect of alcohol have not been fully defined, but some known or suspected mechanisms include (a) alcohol as a solvent for penetration of carcinogens (such as tobacco carcinogens) into mucosal cells, (b) reactive and genotoxic metabolites of alcohol (acetaldehyde), (c) alcohol mediating estrogen and prostaglandin concentration, (d) production of reactive oxygen species and reactive nitrogen species, (e) interactions with DNA repair and folate metabolism, and (f) diets of high alcohol consumers being relatively low in essential nutrients [34].

The recognition of the effects of alcohol consumption on the abundance and composition of gut microbiota came from animal studies. With respect to control mice, mice fed alcohol for 3 weeks were found to have overgrowth of intestinal bacterial, a relative abundance of Bacteroidetes and Verrucomicrobia bacteria and a relative disadvantage of Firmicutes bacteria [35]. In contrast, through metagenomic analysis of the gut microbiome from fecal samples of mice fed alcohol for 6 weeks, chronic ethanol feeding induced a reduction in the abundance of both Bacteroidetes and Firmicutes phyla, with a proportional raise in the Gram-negative Proteobacteria and the Gram-positive Actinobacteria phyla [36]. Nowadays, the correlation between alcohol consumption and changes in gut microbiota in human studies is rare. Bode et al. [37] reported that compared with healthy subjects, chronic alcoholic patients showed a higher number of aerobic and anaerobic microorganisms by examining bacteria in aspirates from the jejunum. Mutlu et al. [38] found that colonic biopsy samples from alcoholics with dysbiosis had lower median abundances of Bacteroidetes and higher ones of Proteobacteria. Similarly, a reduced proportion of Bacteroidetes and highly enriched Proteobacteria and Fusobacteria were found in the alcohol-related cirrhosis group, but similar fecal microbial communities 
was also found in the HBV-related cirrhosis group, which may make one suppose that changes in the fecal microbiota composition were mostly due to cirrhosis [39]. Based on the result that Prevotellaceae was enriched in alcoholic cirrhosis patients with respect to HBV-related cirrhosis patients, the authors speculated that the enrichment of Prevotellaceae may be connected with ethanol metabolism in the human gut [39]. The reason why alcohol consumption could change gut microbiota composition is not completely understood. Some studies proposed that alcohol may affect the intestinal microbiota by reducing gastrointestinal motility, by suppressing innate immune response and adaptive immune response or by inhibiting bactericidal protein expression [40]. Based on these studies, it is difficult to elaborate the influences of alcohol on the intestinal microbiota due to the small sample sizes or different analytical methods used. More advanced knowledge about the mechanisms underlying these findings is needed.

In summary, alcohol intake appears to be a convincing risk factor for CRC. It is recommended to minimize alcohol intake, especially for individuals with high consumption.

\section{Conclusions}

Diet and lifestyle are critical factors for the composition of the gut microbiota. Their interactions with bacterial metabolism also affect not only host metabolism but also commensal bacteria metabolism in the gut lumen. Many microbial metabolites formed from dietary compounds, such as acetaldehyde from ethanol or ammonia, polyamines and hydrogen sulfide from protein, have a role in the initiation and/or progression of CRC, while SCFAs formed from dietary fiber could exert mainly anti-carcinogenic properties. These interactions would also affect immune responses, and it is a well acknowledged that chronic inflammation is a risk factor for CRC. High consumption of fiber, low red or processed red meat intake and minimized alcohol intake would protect against CRC, partly through modulating the gut microbiota. However, there are many interindividual variations in microbial community, and it is difficult to define whether those states of the community are beneficial or bad for one's long-term health. A better theoretical understanding of the influences of diet or lifestyle on the microbial metabolome of individuals is needed. As an easily modifiable environmental factor, it may be possible that applying dietary or lifestyle intervention combined with therapeutic microbiota transplantation could effectively protect against the development of CRC in the future.

\section{Acknowledgments}

This work was funded by the National Natural Science Foundation of China (No. 81572303 to Y.-X. Chen), the National Key Technology R\&D Program (No. 2014BAI09B05 to Y.-X. Chen) and Program of Shanghai Subject Chief Scientist (No. 15XD1502600 to Y.-X. Chen).

\section{Disclosure Statement}

All authors have no personal interests to disclose. 


\section{References}

1 Ferlay J, Soerjomataram I, Dikshit R, Eser S, Mathers C, Rebelo M, Parkin DM, Forman D, Bray F: Cancer incidence and mortality worldwide: sources, methods and major patterns in GLOBOCAN 2012. Int J Cancer 2015; 136:E359-E386.

-2 Song M, Garrett WS, Chan AT: Nutrients, foods, and colorectal cancer prevention. Gastroenterology 2015; 148: 1244-1260.e16.

-3 Wu GD, Chen J, Hoffmann C, Bittinger K, Chen YY, Keilbaugh SA, Bewtra M, Knights D, Walters WA, Knight R, Sinha R, Gilroy E, Gupta K, Baldassano R, Nessel L, Li H, Bushman FD, Lewis JD: Linking long-term dietary patterns with gut microbial enterotypes. Science 2011;334:105-108.

-4 Feng Q, Liang S, Jia H, Stadlmayr A, Tang L, Lan Z, Zhang D, Xia H, Xu X, Jie Z, Su L, Li X, Li X, Li J, Xiao L, HuberSchonauer U, Niederseer D, Xu X, Al-Aama JY, Yang H, Wang J, Kristiansen K, Arumugam M, Tilg H, Datz C, Wang J: Gut microbiome development along the colorectal adenoma-carcinoma sequence. Nat Commun 2015;6: 6528.

5 Ahn J, Sinha R, Pei ZH, Dominianni C, Wu J, Shi JX, Goedert JJ, Hayes RB, Yang LY: Human gut microbiome and risk for colorectal cancer. J Natl Cancer Inst 2013;105:1907-1911.

-6 David LA, Maurice CF, Carmody RN, Gootenberg DB, Button JE, Wolfe BE, Ling AV, Devlin AS, Varma Y, Fischbach MA, Biddinger SB, Dutton RJ, Turnbaugh PJ: Diet rapidly and reproducibly alters the human gut microbiome. Nature 2014;505:559-563.

7 World Cancer Research Fund/American Institute for Cancer Research: Continuous Update Project Report: Keeping the Science Current. Colorectal Cancer 2011 Report: Food, Nutrition, Physical Activity, and the Prevention of Colorectal Cancer. Available at http://www.wcrf.org/sites/default/files/Colorectal-Cancer2011-Report.pdf (accessed October 10, 2015).

-8 Bingham SA, Day NE, Luben R, Ferrari P, Slimani N, Norat T, Clavel-Chapelon F, Kesse E, Nieters A, Boeing H, Tjønneland A, Overvad K, Martinez C, Dorronsoro M, Gonzalez CA, Key TJ, Trichopoulou A, Naska A, Vineis P, Tumino R, Krogh V, Bueno-de-Mesquita HB, Peeters PH, Berglund G, Hallmans G, Lund E, Skeie G, Kaaks R, Riboli E; European Prospective Investigation into Cancer and Nutrition: Dietary fibre in food and protection against colorectal cancer in the European Prospective Investigation into Cancer and Nutrition (EPIC): an observational study. Lancet 2003;361:1496-1501.

\9 Schatzkin A, Mouw T, Park Y, Subar AF, Kipnis V, Hollenbeck A, Leitzmann MF, Thompson FE: Dietary fiber and whole-grain consumption in relation to colorectal cancer in the NIH-AARP Diet and Health Study. Am J Clin Nutr 2007;85:1353-1360.

10 Dahm CC, Keogh RH, Spencer EA, Greenwood DC, Key TJ, Fentiman IS, Shipley MJ, Brunner EJ, Cade JE, Burley VJ, Mishra G, Stephen AM, Kuh D, White IR, Luben R, Lentjes MA, Khaw KT, Rodwell Bingham SA: Dietary fiber and colorectal cancer risk: a nested case-control study using food diaries. J Natl Cancer Inst 2010;102:614626.

11 Ben Q, Sun Y, Chai R, Qian A, Xu B, Yuan Y: Dietary fiber intake reduces risk for colorectal adenoma: a metaanalysis. Gastroenterology 2014;146:689-699.e6.

12 Kunzmann AT, Coleman HG, Huang WY, Kitahara CM, Cantwell MM, Berndt SI: Dietary fiber intake and risk of colorectal cancer and incident and recurrent adenoma in the Prostate, Lung, Colorectal, and Ovarian Cancer Screening Trial. Am J Clin Nutr 2015;102:881-890.

13 Threapleton DE, Greenwood DC, Evans CE, Cleghorn CL, Nykjaer C, Woodhead C, Cade JE, Gale CP, Burley VJ: Dietary fibre intake and risk of cardiovascular disease: systematic review and meta-analysis. BMJ 2013; 347:f6879.

14 Schwiertz A, Taras D, Schäfer K, Beijer S, Bos NA, Donus C, Hardt PD: Microbiota and SCFA in lean and overweight healthy subjects. Obesity (Silver Spring) 2010;18:190-195.

15 Nieuwdorp M, Gilijamse PW, Pai N, Kaplan LM: Role of the microbiome in energy regulation and metabolism. Gastroenterology 2014;146:1525-1533.

16 Encarnação JC, Abrantes AM, Pires AS, Botelho MF: Revisit dietary fiber on colorectal cancer: butyrate and its role on prevention and treatment. Cancer Metastasis Rev 2015;34:465-478.

$>17$ Walker AW, Ince J, Duncan SH, Webster LM, Holtrop G, Ze XL, Brown D, Stares MD, Scott P, Bergerat A, Louis P, McIntosh F, Johnstone AM, Lobley GE, Parkhill J, Flint HJ: Dominant and diet-responsive groups of bacteria within the human colonic microbiota. ISME J 2011;5:220-230.

18 De Filippo C, Cavalieri D, Di Paola M, Ramazzotti M, Poullet JB, Massart S, Collini S, Pieraccini G, Lionetti P: Impact of diet in shaping gut microbiota revealed by a comparative study in children from Europe and rural Africa. Proc Natl Acad Sci USA 2010;107:14691-14696.

19 Albenberg LG, Wu GD: Diet and the intestinal microbiome: associations, functions, and implications for health and disease. Gastroenterology 2014;146:1564-1572.

20 Joshi AD, Kim A, Lewinger JP, Ulrich CM, Potter JD, Cotterchio M, Le Marchand L, Stern MC: Meat intake, cooking methods, dietary carcinogens, and colorectal cancer risk: findings from the Colorectal Cancer Family Registry. Cancer Med 2015;4:936-952.

-21 Parr CL, Hjartåker A, Lund E, Veierød MB: Meat intake, cooking methods and risk of proximal colon, distal colon and rectal cancer: the Norwegian Women and Cancer (NOWAC) cohort study. Int J Cancer 2013;133: 1153-1163. 
22 Bernstein AM, Song M, Zhang X, Pan A, Wang M, Fuchs CS, Le N, Chan AT, Willett WC, Ogino S, Giovannucci EL, $\mathrm{Wu}$ K: Processed and unprocessed red meat and risk of colorectal cancer: analysis by tumor location and modification by time. PLoS One 2015;10:e0135959.

23 Xu XD, Yu ED, Gao XH, Song N, Liu LJ, Wei XB, Zhang W, Fu CG: Red and processed meat intake and risk of colorectal adenomas: a meta-analysis of observational studies. Int J Cancer 2013;132:437-448.

-24 Chan DSM, Lau R, Aune D, Vieira R, Greenwood DC, Kampman E, Norat T: Red and processed meat and colorectal cancer incidence: meta-analysis of prospective studies. PLoS One 2011;6:e20456.

25 McCullough ML, Gapstur SM, Shah R, Jacobs EJ, Campbell PT: Association between red and processed meat intake and mortality among colorectal cancer survivors. J Clin Oncol 2013;31:2773-2782.

-26 Bastide NM, Chenni F, Audebert M, Santarelli RL, Tache S, Naud N, Baradat M, Jouanin I, Surya R, Hobbs DA, Kuhnle GG, Raymond-Letron I, Gueraud F, Corpet DE, Pierre FH: A central role for heme iron in colon carcinogenesis associated with red meat intake. Cancer Res 2015;75:870-879.

27 Demeyer D, Mertens B, De Smet S, Ulens M: Mechanisms linking colorectal cancer to the consumption of (processed) red meat: a review. Crit Rev Food Sci Nutr 2015, Epub ahead of print.

-28 Le Marchand L, Wilkens LR, Kolonel LN, Hankin JH, Lyu LC: Associations of sedentary lifestyle, obesity, smoking, alcohol use, and diabetes with the risk of colorectal cancer. Cancer Res 1997;57:4787-4794.

29 Smyth A, Teo KK, Rangarajan S, O’Donnell M, Zhang X, Rana P, Leong DP, Dagenais G, Seron P, Rosengren A, Schutte AE, Lopez-Jaramillo P, Oguz A, Chifamba J, Diaz R, Lear S, Avezum A, Kumar R, Mohan V, Szuba A, Wei L, Yang W, Jian B, McKee M, Yusuf S; PURE Investigators: Alcohol consumption and cardiovascular disease, cancer, injury, admission to hospital, and mortality: a prospective cohort study. Lancet 2015, Epub ahead of print.

30 Bagnardi V, Rota M, Botteri E, Tramacere I, Islami F, Fedirko V, Scotti L, Jenab M, Turati F, Pasquali E, Pelucchi C, Galeone C, Bellocco R, Negri E, Corrao G, Boffetta P, La Vecchia C: Alcohol consumption and site-specific cancer risk: a comprehensive dose-response meta-analysis. Br J Cancer 2015;112:580-593.

-31 Klarich DS, Brasser SM, Hong MY: Moderate alcohol consumption and colorectal cancer risk. Alcohol Clin Exp Res 2015;39:1280-1291.

-32 Bagnardi V, Rota M, Botteri E, Tramacere I, Islami F, Fedirko V, Scotti L, Jenab M, Turati F, Pasquali E, Pelucchi C, Bellocco R, Negri E, Corrao G, Rehm J, Boffetta P, La Vecchia C: Light alcohol drinking and cancer: a metaanalysis. Ann Oncol 2013;24:301-308.

33 Cho S, Shin A, Park SK, Shin HR, Chang SH, Yoo KY: Alcohol drinking, cigarette smoking and risk of colorectal cancer in the Korean Multi-center Cancer Cohort. J Cancer Prev 2015;20:147-152.

34 Boffetta P, Hashibe M: Alcohol and cancer. Lancet Oncol 2006;7:149-156.

-35 Yan AW, Fouts DE, Brandl J, Starkel P, Torralba M, Schott E, Tsukamoto H, Nelson KE, Brenner DA, Schnabl B: Enteric dysbiosis associated with a mouse model of alcoholic liver disease. Hepatology 2011;53:96-105.

-36 Bull-Otterson L, Feng W, Kirpich I, Wang Y, Qin X, Liu Y, Gobejishvili L, Joshi-Barve S, Ayvaz T, Petrosino J, Kong M, Barker D, McClain C, Barve S: Metagenomic analyses of alcohol induced pathogenic alterations in the intestinal microbiome and the effect of Lactobacillus rhamnosus GG treatment. PLoS One 2013;8:e53028.

-37 Bode JC, Bode C, Heidelbach R, Dürr HK, Martini GA: Jejunal microflora in patients with chronic alcohol abuse. Hepatogastroenterology 1984;31:30-34.

-38 Mutlu EA, Gillevet PM, Rangwala H, Sikaroodi M, Naqvi A, Engen PA, Kwasny M, Lau CK, Keshavarzian A: Colonic microbiome is altered in alcoholism. Am J Physiol Gastrointest Liver Physiol 2012;302:G966-G978.

-39 Chen Y, Yang F, Lu H, Wang B, Chen Y, Lei D, Wang Y, Zhu B, Li L: Characterization of fecal microbial communities in patients with liver cirrhosis. Hepatology 2011;54:562-572.

-40 Vassallo G, Mirijello A, Ferrulli A, Antonelli M, Landolfi R, Gasbarrini A, Addolorato G: Review article: Alcohol and gut microbiota - the possible role of gut microbiota modulation in the treatment of alcoholic liver disease. Aliment Pharmacol Ther 2015;41:917-927. 